\title{
Onyx Embolization of an Acute Type II Endoleak Causing Recurrent Hemorrhage Post Emergency Endovascular Aortic Aneurysm Repair for Ruptured Abdominal Aortic Aneurysm
}

\author{
Justin Pugh ${ }^{1}$ John R. Asquith ${ }^{1}$ Shuvro H. Roy Choudhury ${ }^{1}$ Richard Morgan ${ }^{2}$ David R. Wells ${ }^{1}$ \\ ${ }^{1}$ Department of Interventional Radiology, University Hospitals of \\ North Midlands, Staffordshire, United Kingdom \\ 2Department of Vascular Surgery, University Hospitals of North \\ Midlands, Staffordshire, United Kingdom \\ Address for correspondence Justin Pugh, MBChB, Department of \\ Interventional Radiology, University Hospitals of North Midlands, \\ Stoke-On-Trent, Staffordshire, United Kingdom \\ (e-mail: Justin.pugh@uhns.nhs.uk).
}
Abstract
Keywords
- abdominal aortic aneurysm
- acute type ii endoleak
- endovascular repair

The authors report a case of ongoing retroperitoneal hemorrhage from a ruptured abdominal aortic aneurysm (AAA) following treatment by endovascular abdominal aortic aneurysm repair (EVAR). Unusually, the continued hemorrhage was secondary to a lumbar type II endoleak. This was successfully embolized with onyx. Only one other similar case has been reported.

\section{Introduction}

The overall mortality rate for ruptured abdominal aortic aneurysm (AAA) is high, in excess of $80 \%$, and it remains one of the most common vascular emergencies. ${ }^{1-3}$ The perioperative mortality for emergency open surgical AAA repair also remains high, at more than 40\%. ${ }^{2,3}$ The recent IMPROVE (Immediate Management of the Patient with Ruptured Aneurysm: Open Versus Endovascular Repair) trial demonstrated similar perioperative mortality for endovascular abdominal aortic aneurysm repair (EVAR) compared with open surgical repair of ruptured AAA. ${ }^{2}$ This trial also reported that EVAR is cost-effective, facilitated patient discharge, and improved health-related quality of life compared with open surgery for rupture. ${ }^{3}$ Consequently, EVAR has become the preferred treatment option for anatomically suitable ruptured AAA in many institutions.

However, EVAR has potential complications, of which type II endoleak is the most common. Type II endoleak occurs when retrograde blood flow from aortic branches leads to continued perfusion of the AAA sac. ${ }^{4}$ Type II endoleaks occur in 10 to $44 \%$ of EVARs and do not usually cause sac expansion. Type II endoleaks are not always benign. Over the long term, they can produce increased aneurysm sac size and subsequent AAA rupture. ${ }^{4-6}$ Although the authors have observed type II endoleaks after rupture EVAR, they have until now been without acute complication. The authors describe an unusual case of early recurrent retroperitoneal hemorrhage secondary to a lumbar type II endoleak post rupture EVAR.

\section{Case Report}

A 71-year-old man was admitted to the emergency department with acute abdominal pain radiating to the left side and back. His blood pressure was $137 / 74 \mathrm{~mm} \mathrm{Hg}$, heart rate 82 beats $/ \mathrm{min}$, and hemoglobin of $11.5 \mathrm{~g} / \mathrm{dL}$. Urgent computed tomography angiography (CTA) demonstrated a ruptured $93 \mathrm{~mm}$ diameter infrarenal AAA, with active contrast extravasation from a $16-\mathrm{mm}$ defect in the left posterolateral wall of the AAA and a large retroperitoneal hematoma ( - Fig. 1). The aneurysm was anatomically suitable for EVAR, with a neck diameter of $25 \mathrm{~mm}$, length of $19 \mathrm{~mm}$, and no significant angulation. Common iliac artery diameters on the left of $19 \mathrm{~mm}$, right of $15 \mathrm{~mm}$, and suitable length iliac landing zones were noted.

An emergency EVAR was performed under general anesthetic, using a bifurcated Endurant II graft (Medtronic). The graft juxtaposed the renal arteries superiorly and the origins of the internal iliac arteries inferiorly. A completion intraoperative angiogram showed good positioning of the graft, exclusion of the aneurysm sac, and no evidence of significant endoleak ( - Fig. 2). The procedure lasted $90 \mathrm{~min}$ utes, during which the patient required 3 units of red blood received

June 9, 2017

accepted after revision

September 27, 2017
DOI https://doi.org/

10.1055/s-0038-1641677.

ISSN 2457-0214.
Copyright $\odot 2018$ by Indian Society of Vascular and Interventional Radiology
License terms

() (1) $\Theta \circledast$ 


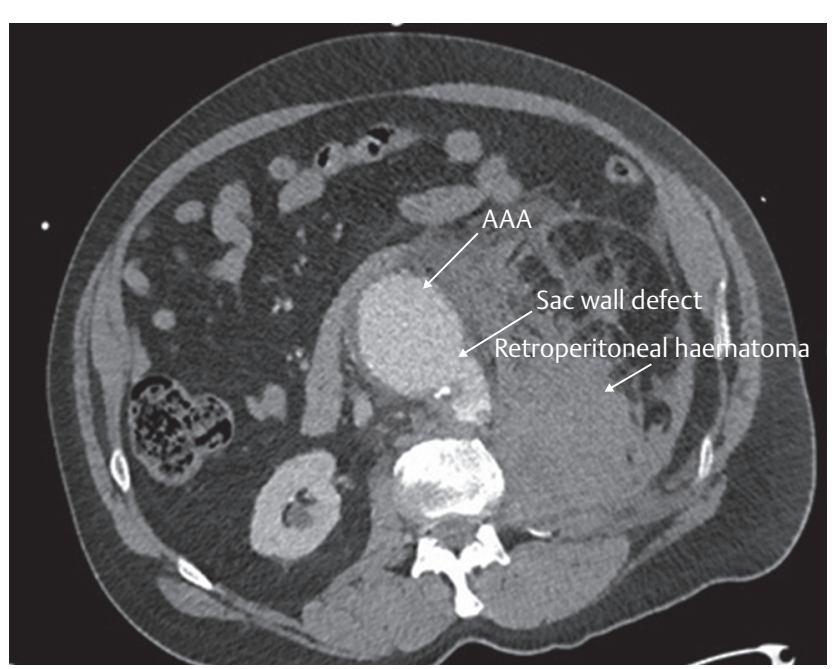

Fig. 1 Axial CTA image showing aneurysm wall defect with active contrast extravasation and resultant retroperitoneal hematoma.

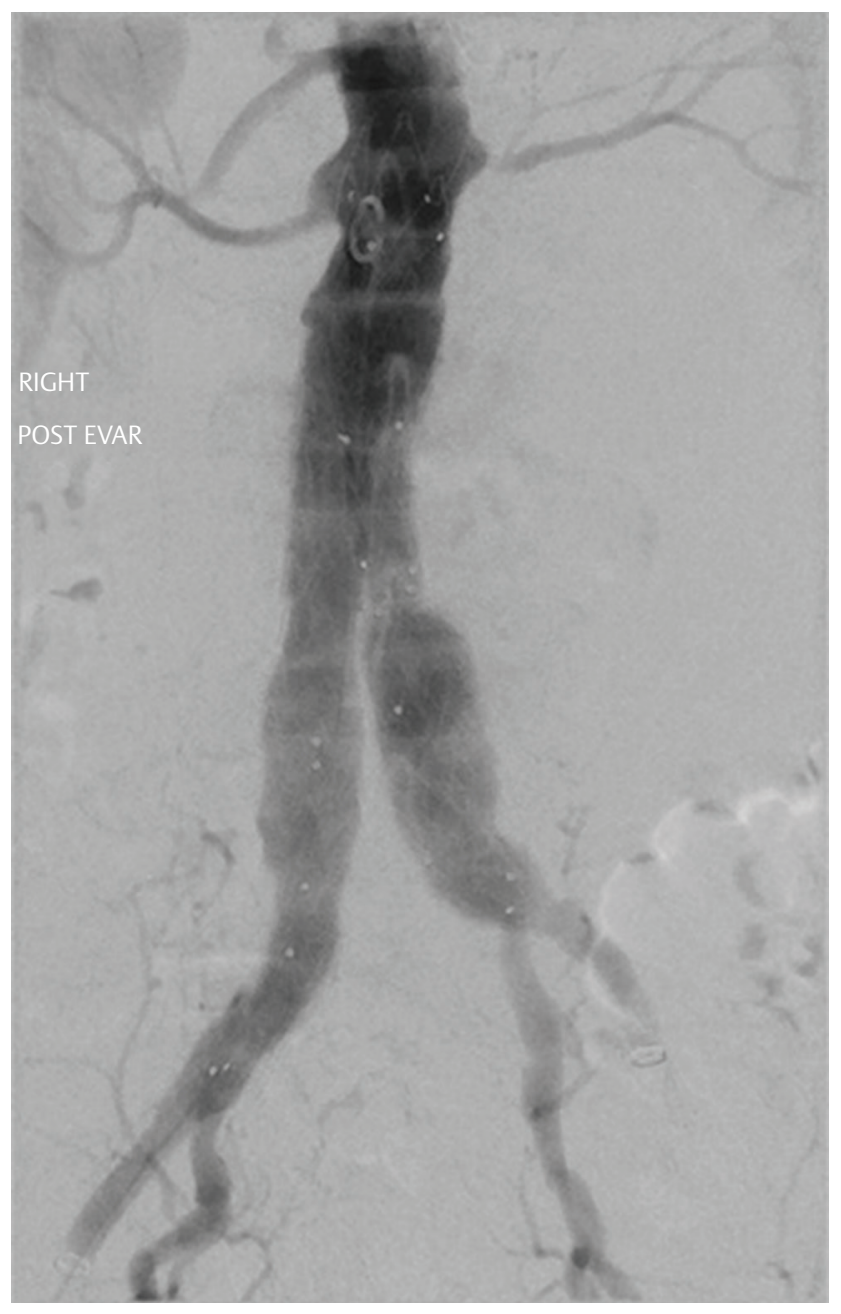

Fig. 2 Completion digital subtraction angiography post EVAR. Imaging suggests exclusion of the aneurysm sac without significant endoleak, with satisfactory graft position.

cells, 2 units of fresh frozen plasma, and inotropic support (metaraminol).

Post EVAR, the patient was monitored in intensive care and initially showed signs of improvement. However, over the course of the next 2 days, he had recurring abdominal pain, abdominal distension, a drop of hemoglobin to $6.8 \mathrm{~g} /$ $\mathrm{dL}$, and became cardiovascularly compromised. A repeat CTA showed an increased size of the retroperitoneal hematoma secondary to a lumbar type II endoleak in continuity with a tract of contrast extending through the original aneurysm wall defect ( $\boldsymbol{- \text { Fig. }} \mathbf{3}$ ). The scan confirmed good position of the EVAR stent graft and no type I or III endoleak.

The patient was transferred immediately to Interventional Radiology for further angiography. An 8F sheath (Cordis) was inserted on the right for access to the right iliolumbar artery and a 14F sheath (Medtronic) on the left should balloon occlusion of the aorta be required for emergency blood pressure control. Aortography showed no type I or III endoleaks. Selective angiography of the right iliolumbar artery was performed using a 4F Sim 1 (Cordis) catheter and Progreat microcatheter (Terumo). This confirmed a lumbar type II endoleak in continuity with extravasation of contrast outside the AAA sac. Cannulation of the endoleak via the right iliolumbar artery failed due to the small caliber and tortuous path of the right iliolumbar artery.

The endoleak nidus was reached via a left-sided transiliac paraendograft approach ( - Fig. 4). A 4F Berenstein catheter (Cordis) and stiff hydrophilic guidewire (Boston Scientific) were passed between the left iliac graft and adjacent vessel wall. A Progreat microcatheter (Terumo) was then advanced into the endoleak nidus within the aneurysm sac. The nidus and its associated feeding lumbar arteries were successfully embolized with $4.3 \mathrm{~mL}$ Onyx (eV3 Neurovascular) (-Fig. 5). Once embolization took place, the patient immediately stabilized and no longer required inotropic support. Completion angiography demonstrated no further endoleaks. The patient was transferred to intensive care and had an uneventful recovery, being moved to the vascular ward 2 days later and then discharged home 8 days later.

Follow-up CTA at 1 and 6 months post-EVAR showed reduction in size of the aneurysm sac to $89 \mathrm{~mm}$ and $75 \mathrm{~mm}$, respectively. The type II endoleak and feeding lumbar arteries remained occluded. There was also significant reduction in the size of the retroperitoneal hematoma at 1 month and near-complete resolution at 6 months.

\section{Discussion}

The aim of EVAR in the acute rupture setting is to exclude the aneurysm sac and control blood loss. Endoleak is the persistent perfusion of the aneurysm sac post EVAR, ${ }^{7}$ with a reported incidence varying from 15 to $52 \%$, that can lead to sac pressurization, with continued rupture risk. ${ }^{5,6}$ The most common endoleaks post EVAR are type II, occurring in 10 to $44 \%$ of patients. ${ }^{4-6,8,9}$ Type II endoleaks can be classified as transient or persistent, lasting less or more than 6 months respectively. ${ }^{9}$ Early and late type II endoleaks occur within 30 days or over 1 year post EVAR, respectively. ${ }^{4}$ Lumbar and inferior mesenteric arteries are most frequently involved. Type II endoleaks form a nidus akin to a vascular lake within the sac thrombus, with the nidus having similar properties to a vascular malformation. ${ }^{4,9}$ 

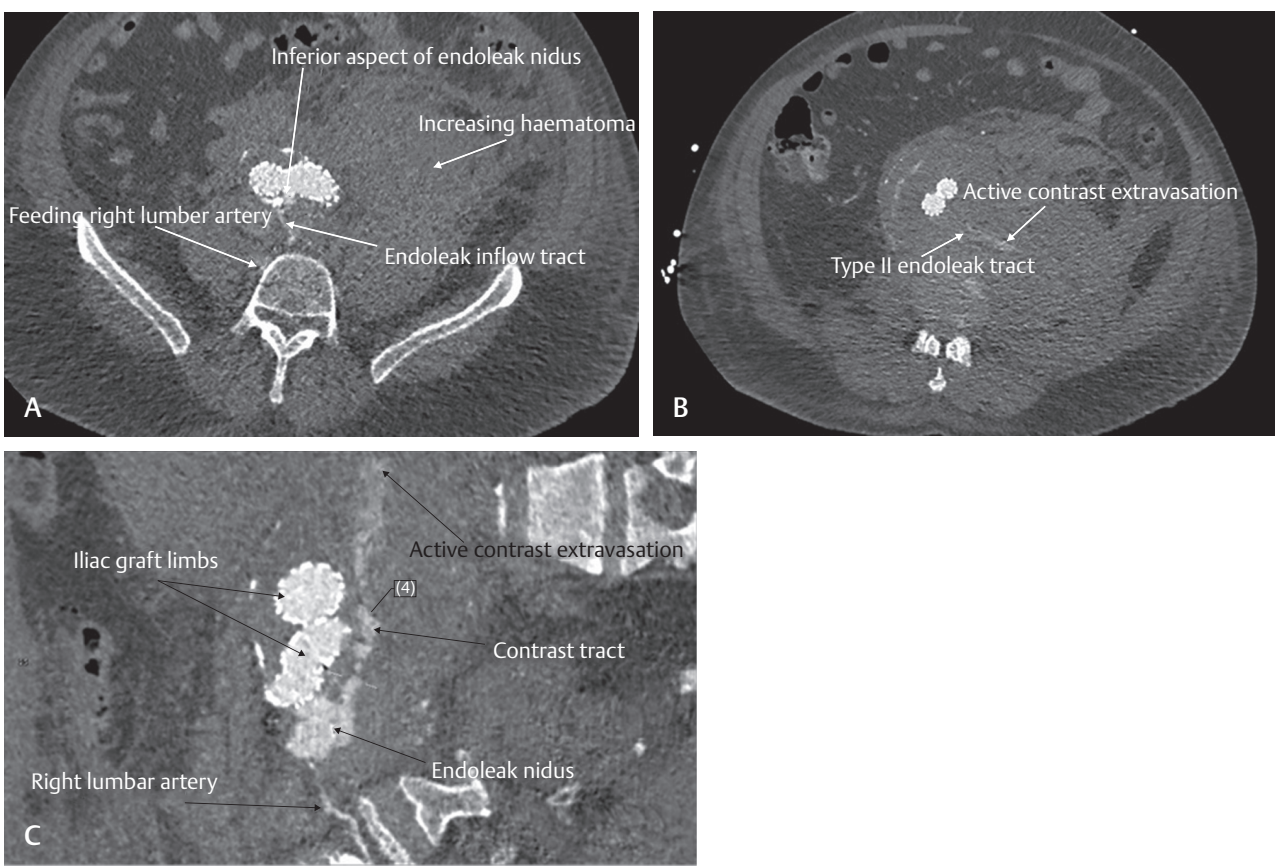

Fig. 3 Axial CTA images (A, B) demonstrate endoleak inflow to the nidus via the right lumbar artery, nidus, and outflow tract to the sac wall defect with resultant contrast extravasation and increasing hematoma size. Curved MPR image (C) shows the features a little better, although with somewhat distorted views.

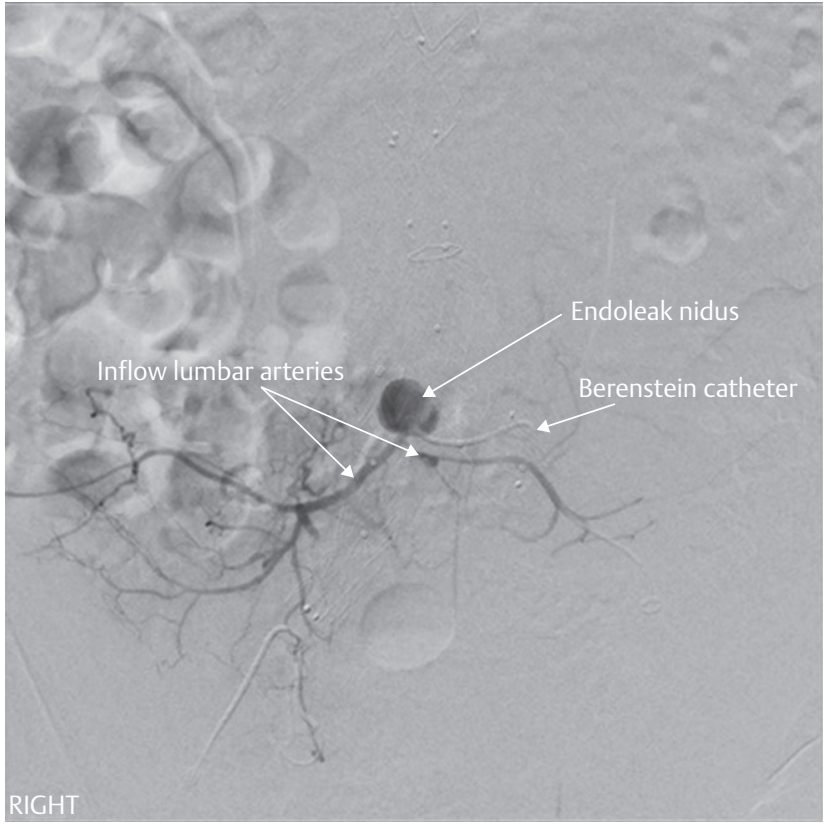

Fig. 4 Digital subtraction angiography demonstrating inflow arteries and endoleak nidus. Extravasation blush was demonstrated on fluoro loop and sadly not stored.

Uncomplicated persistent type II endoleaks are frequently treated conservatively with follow up imaging. Persistent type II endoleaks that are symptomatic or demonstrate sac size increases of greater than $5 \mathrm{~mm}$ generally undergo secondary intervention. Embolization of both the nidus and the feeding artery is desirable to reduce endoleak recurrence, ${ }^{8,9}$ and this is theoretically best achieved with liquid embolic agents. ${ }^{9}$ The preferred method of management for this in our institution is transarterial embolization of both the nidus

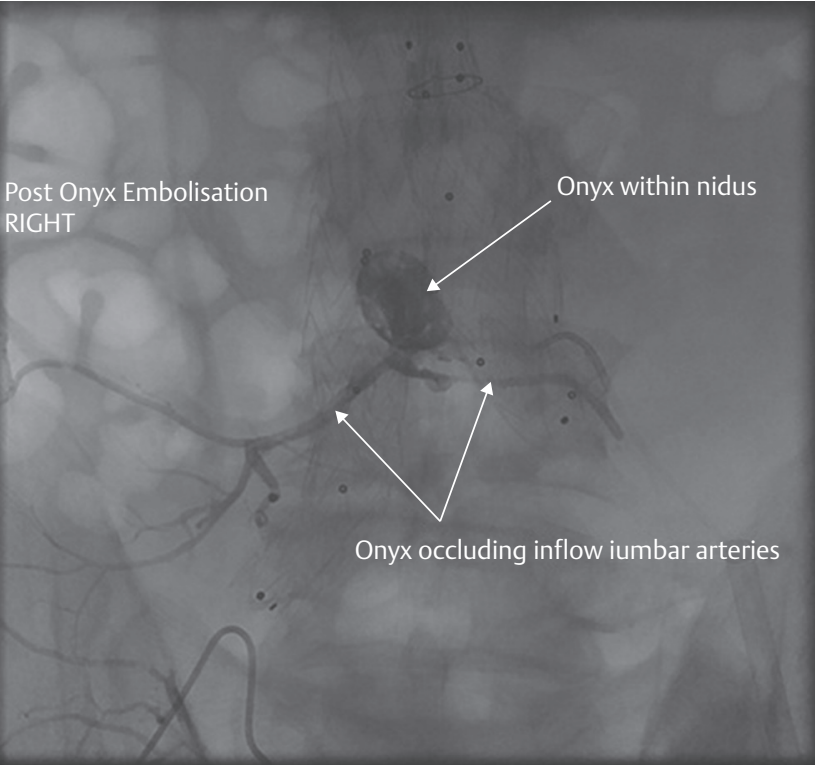

Fig. 5 Digital subtraction angiography demonstrates opacification of the endoleak nidus and inflow lumbar arteries with onyx.

and the feeding vessels with Onyx. In this case, a transiliac paraendograft approach was easily performed, presumably because the EVAR had only been performed 2 days previously.

Early type II endoleak post rupture EVAR with persisting blood loss and increasing retroperitoneal hematoma is a rare phenomenon, having been reported once before in the literature. ${ }^{10}$ The authors have never previously encountered this complication despite often observing uncomplicated early type II endoleaks in the rupture EVAR setting. In the previously described case report, the vessel feeding the endoleak was treated by coil embolization. In this patient, angiography 
on completion of the EVAR was unremarkable and showed no evidence of endoleak. However, there was continued hemorrhage, retroperitoneal hematoma growth, and cardiovascular compromise. This was shown to be due to type II endoleak with active contrast extravasation from the original aneurysm wall defect. This necessitated urgent treatment of the endoleak. There was successful embolization of the endoleak with onyx, with almost immediate cessation of required inotropic support.

\section{Conclusion}

Although frequently benign, early type II endoleak post rupture EVAR may rarely cause continued aneurysm leak and should be considered as a potential etiology of ongoing aneurysm hemorrhage. The authors successfully managed such a case with onyx embolization.

\section{Conflict of Interest}

None.

\section{References}

1 Karthikesalingam A, Holt PJ, Vidal-Diez A, et al. Mortality from ruptured abdominal aortic aneurysms: clinical lessons from a comparison of outcomes in England and the USA. Lancet 2014;383(9921):963-969

2 Powell JT, Sweeting MJ, Thompson MM, et al; IMPROVE Trial Investigators. Endovascular or open repair strategy for ruptured abdominal aortic aneurysm: 30 day outcomes from IMPROVE randomised trial. BMJ 2014;348:f7661
3 Grieve R, Gomes M, Sweeting MJ, et al; IMPROVE Trial Investigators. Endovascular strategy or open repair for ruptured abdominal aortic aneurysm: one-year outcomes from the IMPROVE randomized trial. Eur Heart J 2015;36(31):2061-2069

4 Brown A, Saggu GK, Bown MJ, Sayers RD, Sidloff DA. Type II endoleaks: challenges and solutions. Vasc Health Risk Manag 2016;12:53-63

5 Kaczynski J, Jaber B, Woolgar J. Rupture of the infrarenal abdominal aortic aneurysm (AAA) following an endovascular aneurysm repair (EVAR) due to an isolated type II endoleak. BMJ Case Rep 2014;2014(14):bcr2013202964

6 Chan KK, Siu WT, Fung KH, Yau KK, Wong SK, Li MK. Acute symptomatic abdominal aortic aneurysm secondary to endovascular stent graft associated type II endoleak. Asian J Surg 2006;29(3):157-160

7 Khaja MS, Park AW, Swee W, et al. Treatment of type II endoleak using onyx with long-term imaging follow-up. Cardiovasc Intervent Radiol 2014;37(3):613-622

8 Saqib NU, Charlton-Ouw KM, Azizzadeh A. Managing type II endoleaks. Endovasc Today 2013:45-50

9 Chung R, Morgan RA. Type 2 endoleaks post-EVAR: current evidence for rupture risk, intervention and outcomes of treatment. Cardiovasc Intervent Radiol 2015;38(3):507-522

10 Hartung O, Vidal V, Marani I, Saran A, Bartoli JM, Alimi YS. Treatment of an early type II endoleak causing hemorrhage after endovascular aneurysm repair for ruptured abdominal aortic aneurysm. J Vasc Surg 2007;45(5):1062-1065 